\title{
COMPARISONS OF THE EXACT AND THE APPROXIMATE SOLUTIONS OF SECOND-ORDER FUZZY LINEAR BOUNDARY VALUE PROBLEMS
}

\author{
HÜLYA GÜLTEKIN ÇITIL
}

Received 10 May, 2018

\begin{abstract}
In this paper, the approximate solutions by using the undetermined fuzzy coefficients method and the exact solutions by using the Hukuhara differentiability of second-order fuzzy linear boundary value problems with constant coefficients are investigated. Thus, comparisons of the found solutions are given.
\end{abstract}

2010 Mathematics Subject Classification: 03E72; 34A07; 34B05

Keywords: fuzzy boundary value problems, second-order fuzzy differential equation, Hukuhara differentiability, fuzzy approximate solution

\section{INTRODUCTION}

Many researchers study fuzzy differential equations. Because, fuzzy differential equations form the mathematical model of real world problems in which uncertainty. Fuzzy differential equations can be solved with several approach. The first approach is Hukuhara differentiability. Firstly, the existence and uniqueness of the solution of fuzzy differential equation were examined [7,14]. The second approach is generalized differentiability. Khastan and Nieto [15] found new solutions for some fuzzy boundary value problems using the generalized differentiability. Using the generalized differentiability, they found solutions for larger class of fuzzy boundary value problems than using the Hukuhara differentiability. The third approach generate the fuzzy solution from the crips solution. This can be three ways. The first one is extension principle and here the initial value is taken as a real constant, crips problem is solved and then the real constant in the solution is replaced with the fuzzy initial value [7, 8]. The second way is the concept of differential inclusion [13]. Here, the alpha-cut of the initial value is taken and the differential equation is converted to a differential inclusion. The third way consider the fuzzy problem to be a set of crips problem [10]. Howewer, many fuzzy initial and boundary value problems can not be solved as analytical every time. Thus, to find approximate solutions of these 
problems is important. The numeric methods were introduced in [1,2,4]. Allahviranloo [3] found the approximate solution of nth-order linear differential equations with fuzzy initial conditions by the collocation method. Guo at al. [11] found the approximate solution of a class of-second-order linear differential equation with fuzzy boundary value conditions by the undetermined fuzzy coefficients method.

In this paper, the approximate solutions by using the undetermined fuzzy coefficients method and the exact solutions by using the Hukuhara differentiability of second-order fuzzy linear boundary value problems constant coefficients are investigated . Thus, comparison results of the found solutions are given. The aim of this study is to investigate how close the exact solutions to of the approximate solutions by the undetermined fuzzy coefficients method of fuzzy boundary value problems for fuzzy differential equations with the positive and negative constant coefficients.

\section{Preliminaries}

Definition 1 ([16]). A fuzzy number is a mapping $u: \mathbb{R} \rightarrow[0,1]$ satisfying the following properties:

$\mathrm{u}$ is normal: $\exists x_{0} \in \mathbb{R}$ for which $u\left(x_{0}\right)=1$,

$\mathrm{u}$ is convex fuzzy set: $u(\lambda x+(1-\lambda) y) \geqslant \min \{u(x), u(y)\}$ for all $x, y \in \mathbb{R}, \lambda \in$ $[0,1]$,

$\mathrm{u}$ is upper semi-continuous on $\mathbb{R}$,

$c l\{x \in \mathbb{R} \mid u(x)>0\}$ is compact, where $c l$ denotes the closure of a subset.

Let $\mathbb{R}_{F}$ denote the set of all fuzzy numbers.

Definition 2 ([15]). Let $u \in \mathbb{R}_{F}$. The $\alpha$-level set of $u$, denoted , $[u]^{\alpha}, 0<\alpha \leq 1$, is $[u]^{\alpha}=\{x \in \mathbb{R} \mid u(x) \geq \alpha\}$. If $\alpha=0,[u]^{0}=c l\{$ suppu $\}=c l\{x \in \mathbb{R} \mid u(x)>0\}$. The notation, $[u]^{\alpha}=\left[\underline{u}_{\alpha}, \bar{u}_{\alpha}\right]$ denotes explicitly the $\alpha$-level set of $u$, where $\underline{u}_{\alpha}$ and $\bar{u}_{\alpha}$ denote the left-hand endpoint and the right-hand endpoint of $[u]^{\alpha}$, respectively.

The following remark shows that $\left[\underline{u}_{\alpha}, \bar{u}_{\alpha}\right]$ is a valid $\alpha$-level set.

Remark $1([9,15])$. The sufficient and necessary conditions for $\left[\underline{u}_{\alpha}, \bar{u}_{\alpha}\right]$ to define the parametric form of a fuzzy number as follows:

$\underline{u}_{\alpha}$ is bounded monotonic increasing (non-decreasing) left-continuous function on $(0,1]$ and right-continuous for $\alpha=0$,

$\bar{u}_{\alpha}$ is bounded monotonic decreasing (non-increasing) left-continuous function on $(0,1]$ and right-continuous for $\alpha=0$,

$\underline{u}_{\alpha} \leq \bar{u}_{\alpha}, 0 \leq \alpha \leq 1$.

Definition 3 ([16]). If $\mathrm{A}$ is a symmetric triangular fuzzy number with support $[\underline{a}, \bar{a}]$, the $\alpha$-level set of $A$ is $[A]^{\alpha}=\left[\underline{A}_{\alpha}, \bar{A}_{\alpha}\right]=\left[\underline{a}+\left(\frac{\bar{a}-\underline{a}}{2}\right) \alpha, \bar{a}-\left(\frac{\bar{a}-\underline{a}}{2}\right) \alpha\right]$, $\left(\underline{A}_{1}=\bar{A}_{1}, \underline{A}_{1}-\underline{A}_{\alpha}=\bar{A}_{\alpha}-\bar{A}_{1}\right)$. 
Definition $4([11,12])$. For arbitrary $u=[\underline{u}, \bar{u}], v=[\underline{v}, \bar{v}] \in \mathbb{R}_{F}$, the quantity

$$
D(u, v)=\left(\int_{0}^{1}\left(\underline{u}_{\alpha}-\underline{v}_{\alpha}\right)^{2} d \alpha+\int_{0}^{1}\left(\bar{u}_{\alpha}-\bar{v}_{\alpha}\right)^{2} d \alpha\right)^{\frac{1}{2}}
$$

is the distance between fuzzy numbers $u$ and $v$.

Definition 5 ([11,15,17]). Let $u, v \in \mathbb{R}_{F}$. If there exists $w \in \mathbb{R}_{F}$ such that $u=$ $v+w$, then $w$ is called the Hukuhara difference of fuzzy numbers $u$ and $v$, and it is denoted by $w=u \ominus v$.

Definition $6([5,11,15])$. Let $f:[a, b] \rightarrow \mathbb{R}_{F}$ and $t_{0} \in[a, b]$. We say that $\mathrm{f}$ is Hukuhara differential at $t_{0}$, if there exists an element $f^{\prime}\left(t_{0}\right) \in \mathbb{R}_{F}$ such that for all $h>0$ sufficiently small, $\exists f\left(t_{0}+h\right) \ominus f\left(t_{0}\right), f\left(t_{0}\right) \ominus f\left(t_{0}-h\right)$ and the limits (in the metric D)

$$
\lim _{h \rightarrow 0} \frac{f\left(t_{0}+h\right) \ominus f\left(t_{0}\right)}{h}=\lim _{h \rightarrow 0} \frac{f\left(t_{0}\right) \ominus f\left(t_{0}-h\right)}{h}=f^{\prime}\left(t_{0}\right) .
$$

Lemma 1 ([6]). If $g:[a, b] \rightarrow \mathbb{R}$ is differential on $[a, b]$ such that $g^{\prime}, g^{\prime \prime}$ are non-negative and monotonic increasing on $[a, b]$, then $\forall c \in \mathbb{R}_{F}, f(x)=c g(x)$ is differential on $[a, b]$ and

$$
f^{\prime}(x)=c g^{\prime}(x), f^{\prime \prime}(x)=c g^{\prime \prime}(x), \forall x \in[a, b] .
$$

Definition 7 ([11]). The undetermined fuzzy coefficients method is to seek an approximate solution as

$$
\tilde{y}_{N}(t)=\sum_{k=0}^{N} \tilde{\theta}_{k} \phi_{k}(t),
$$

where, $\phi_{k}(t), k=0,1, \ldots, N$ are positive basic functions whose all differentiations are positive.

Lemma $2([3,11])$. Let the basis functions $\phi_{k}(t), k=0,1, \ldots, N$ and all of their differentiations be positive, without loss of generality. Then $\left(\underline{y}_{N}\right)^{(i)}(t)=\underline{y}_{N}^{(i)}(t)$ and $\left(\bar{y}_{N}^{(i)}\right)(t)=\overline{y_{N}^{(i)}}(t), i=0,1,2$.

\section{SECOND-ORDER FUZZY LINEAR BOUNDARY VALUE PROBLEMS}

3.1. The case of positive constant coefficient

Consider the fuzzy boundary value problem

$$
\begin{aligned}
& y^{\prime \prime}(t)=\lambda y(t), t \in[0, \ell] \\
& y(0)=A, \quad y(\ell)=B,
\end{aligned}
$$


where $\lambda>0, \mathrm{~A}$ and $\mathrm{B}$ are symmetric triangular fuzzy numbers with supports $[\underline{a}, \bar{a}]$ and $[\underline{b}, \bar{b}]$, respectively,

$$
[A]^{\alpha}=\left[\underline{a}+\left(\frac{\bar{a}-\underline{a}}{2}\right) \alpha, \bar{a}-\left(\frac{\bar{a}-a}{2}\right) \alpha\right],[B]^{\alpha}=\left[\underline{b}+\left(\frac{\bar{b}-\underline{b}}{2}\right) \alpha, \bar{b}-\left(\frac{\bar{b}-\underline{b}}{2}\right) \alpha\right] .
$$

\subsubsection{The Exact Solution with Hukuhara Differentiability}

From the fuzzy differential equation (3.1), we have differential equations

$$
\begin{gathered}
\underline{Y}_{\alpha}^{\prime \prime}(t)=\lambda \underline{Y}_{\alpha}(t), \\
\bar{Y}_{\alpha}^{\prime \prime}(t)=\lambda \bar{Y}_{\alpha}(t)
\end{gathered}
$$

by using the Hukuhara differentiability. Then, the lower solution and the upper solution of the fuzzy differential equation (3.1) are obtained as

$$
\begin{gathered}
\underline{Y}_{\alpha}(t)=\underline{c}_{1}(\alpha) e^{\sqrt{\lambda} t}+\underline{c}_{2}(\alpha) e^{-\sqrt{\lambda} t}, \\
\bar{Y}_{\alpha}(t)=\bar{c}_{1}(\alpha) e^{\sqrt{\lambda} t}+\bar{c}_{2}(\alpha) e^{-\sqrt{\lambda} t} .
\end{gathered}
$$

Using the boundary conditions (3.2), coefficients $\underline{c}_{1}(\alpha), \underline{c}_{2}(\alpha), \bar{c}_{1}(\alpha), \bar{c}_{2}(\alpha)$ are solved as

$$
\begin{aligned}
& \underline{c}_{1}(\alpha)=\frac{\left(\underline{b}+\left(\frac{\bar{b}-\underline{b}}{2}\right) \alpha\right)-\left(\underline{a}+\left(\frac{\bar{a}-\underline{a}}{2}\right) \alpha\right) e^{-\sqrt{\lambda \ell}}}{e^{\sqrt{\lambda} \ell}-e^{-\sqrt{\lambda} \ell}}, \\
& \underline{c}_{2}(\alpha)=\frac{\left(\underline{a}+\left(\frac{\bar{a}-\underline{a}}{2}\right) \alpha\right) e^{\sqrt{\lambda \ell}}-\left(\underline{b}+\left(\frac{\bar{b}-\underline{b}}{2}\right) \alpha\right)}{e^{\sqrt{\lambda} \ell}-e^{-\sqrt{\lambda \ell}}}, \\
& \bar{c}_{1}(\alpha)=\frac{\left(\bar{b}-\left(\frac{\bar{b}-\underline{b}}{2}\right) \alpha\right)-\left(\bar{a}-\left(\frac{\bar{a}-a}{2}\right) \alpha\right) e^{-\sqrt{\lambda} \ell}}{e^{\sqrt{\lambda} \ell}-e^{-\sqrt{\lambda} \ell}}, \\
& \bar{c}_{2}(\alpha)=\frac{\left(\bar{a}-\left(\frac{\bar{a}-\underline{a}}{2}\right) \alpha\right) e^{\sqrt{\lambda \ell}}-\left(\bar{b}-\left(\frac{\bar{b}-\underline{b}}{2}\right) \alpha\right)}{e^{\sqrt{\lambda \ell}}-e^{-\sqrt{\lambda \ell}}} .
\end{aligned}
$$

3.1.2. The Approximate Solution with the Undetermined Fuzzy Coefficients Method

An approximate solution with the undetermined fuzzy coefficients method is

$$
\tilde{y}_{N}(t)=\sum_{k=0}^{N} \tilde{\theta}_{k} \phi_{k}(t),
$$

where, $\phi_{k}(t), k=0,1, \ldots, N$ are positive basic functions whose all differentiations are positive and the lower solution and upper solution are

$$
\underline{y}_{\alpha}(t)=\sum_{k=0}^{N} \underline{\theta}_{k}(\alpha) \phi_{k}(t), \quad \bar{y}_{\alpha}(t)=\sum_{k=0}^{N} \bar{\theta}_{k}(\alpha) \phi_{k}(t),
$$


respectively. Substituting the expression (3.3) in the fuzzy differential equation (3.1) yields

$$
\begin{aligned}
& \sum_{k=0}^{N} \underline{\theta}_{k}(\alpha) \phi_{k}^{\prime \prime}(t)-\lambda \sum_{k=0}^{N} \underline{\theta}_{k}(\alpha) \phi_{k}(t)=0, \\
& \sum_{k=0}^{N} \bar{\theta}_{k}(\alpha) \phi_{k}^{\prime \prime}(t)-\lambda \sum_{k=0}^{N} \bar{\theta}_{k}(\alpha) \phi_{k}(t)=0 .
\end{aligned}
$$

Using the boundary conditions (3.2),

$$
\begin{aligned}
& \sum_{k=0}^{N} \underline{\theta}_{k}(\alpha) \phi_{k}(0)=\underline{a}+\left(\frac{\bar{a}-\underline{a}}{2}\right) \alpha, \\
& \sum_{k=0}^{N} \underline{\theta}_{k}(\alpha) \phi_{k}(\ell)=\underline{b}+\left(\frac{\bar{b}-\underline{b}}{2}\right) \alpha, \\
& \sum_{k=0}^{N} \bar{\theta}_{k}(\alpha) \phi_{k}(0)=\bar{a}-\left(\frac{\bar{a}-\underline{a}}{2}\right) \alpha, \\
& \sum_{k=0}^{N} \bar{\theta}_{k}(\alpha) \phi_{k}(\ell)=\bar{b}-\left(\frac{\bar{b}-\underline{b}}{2}\right) \alpha
\end{aligned}
$$

are obtained. Taking

$$
\phi_{k}^{\prime \prime}(t)+\lambda \phi_{k}(t)=\rho_{k}, \quad \phi_{k}(0)=\zeta_{0 k}, \quad \phi_{k}(\ell)=\xi_{\ell k},
$$

we obtain

$$
\begin{gathered}
\sum_{k=0}^{N} \underline{\theta}_{k}(\alpha) \rho_{k}=0, \\
\sum_{k=0}^{N} \underline{\theta}_{k}(\alpha) \zeta_{0 k}=\underline{a}+\left(\frac{\bar{a}-\underline{a}}{2}\right) \alpha, \\
\sum_{k=0}^{N} \underline{\theta}_{k}(\alpha) \xi_{\ell k}=\underline{b}+\left(\frac{\bar{b}-\underline{b}}{2}\right) \alpha, \\
\sum_{k=0}^{N} \bar{\theta}_{k}(\alpha) \rho_{k}=0, \\
\sum_{k=0}^{N} \bar{\theta}_{k}(\alpha) \zeta_{0 k}=\bar{a}-\left(\frac{\bar{a}-\underline{a}}{2}\right) \alpha,
\end{gathered}
$$




$$
\sum_{k=0}^{N} \bar{\theta}_{k}(\alpha) \xi_{\ell k}=\bar{b}-\left(\frac{\bar{b}-\underline{b}}{2}\right) \alpha .
$$

Let us write the equations (3.4) - (3.9) as

$$
S(t) X(\alpha)=Y(\alpha),
$$

where

$$
\begin{aligned}
& S=\left(\begin{array}{ll}
S_{1} & S_{2} \\
S_{2} & S_{1}
\end{array}\right) \\
& S_{1}=\left(\begin{array}{cccc}
\rho_{1} & \rho_{2} & \ldots & \rho_{N} \\
\zeta_{00} & \zeta_{01} & \ldots & \zeta_{0 N} \\
\xi_{\ell 0} & \xi_{\ell 1} & \ldots & \xi_{\ell N}
\end{array}\right), \quad S_{2}=\left(\begin{array}{cccc}
0 & 0 & \ldots & 0 \\
0 & 0 & \ldots & 0 \\
0 & 0 & \ldots & 0
\end{array}\right), \\
& X(\alpha)=\left(\underline{\theta}_{0} \underline{\theta}_{1} \ldots \underline{\theta}_{N} \bar{\theta}_{0} \bar{\theta}_{1} \ldots . \bar{\theta}_{N}\right)^{T}, Y(\alpha)=\left(0 \underline{A}_{\alpha} \underline{B}_{\alpha} 0 \bar{A}_{\alpha} \bar{B}_{\alpha}\right)^{T} .
\end{aligned}
$$

From this, $\underline{\theta}_{0}, \underline{\theta}_{1}, \ldots \underline{\theta}_{N}, \bar{\theta}_{0}, \bar{\theta}_{1}, \ldots, \bar{\theta}_{N}$ are solved and the approximate solution is obtained [11].

Example 1. Consider the fuzzy boundary value problem

$$
\begin{gathered}
y^{\prime \prime}(t)=y(t), t \in\left(0, \frac{3}{2} \pi\right), \\
y(0)=\left[1+\frac{1}{2} \alpha, 2-\frac{1}{2} \alpha\right], y\left(\frac{3 \pi}{2}\right)=\left[3+\frac{1}{2} \alpha, 4-\frac{1}{2} \alpha\right] .
\end{gathered}
$$

Using the Hukuhara differentiability, the lower exact solution and the upper exact solution of the fuzzy differential equation (3.10) are obtained as

$$
\begin{aligned}
& \underline{Y}_{\alpha}(t)=\underline{c}_{1}(\alpha) e^{t}+\underline{c}_{2}(\alpha) e^{-t}, \\
& \bar{Y}_{\alpha}(t)=\bar{c}_{1}(\alpha) e^{t}+\bar{c}_{2}(\alpha) e^{-t} .
\end{aligned}
$$

Using the boundary conditions (3.11),

$$
\begin{aligned}
& \underline{c}_{1}(\alpha)=\frac{\left(3+\frac{1}{2} \alpha\right)-\left(1+\frac{1}{2} \alpha\right) e^{-\frac{3 \pi}{2}}}{e^{\frac{3 \pi}{2}}-e^{-\frac{3 \pi}{2}}} \\
& \underline{c}_{2}(\alpha)=\frac{\left(1+\frac{1}{2} \alpha\right) e^{\frac{3 \pi}{2}}-\left(3+\frac{1}{2} \alpha\right)}{e^{\frac{3 \pi}{2}}-e^{-\frac{3 \pi}{2}}} \\
& \bar{c}_{1}(\alpha)=\frac{\left(4-\frac{1}{2} \alpha\right)-\left(2-\frac{1}{2} \alpha\right) e^{-\frac{3 \pi}{2}}}{e^{\frac{3 \pi}{2}}-e^{-\frac{3 \pi}{2}}} \\
& \bar{c}_{2}(\alpha)=\frac{\left(2-\frac{1}{2} \alpha\right) e^{\frac{3 \pi}{2}}-\left(4-\frac{1}{2} \alpha\right)}{e^{\frac{3 \pi}{2}}-e^{-\frac{3 \pi}{2}}}
\end{aligned}
$$

are obtained. Now, let us find the approximate solution. Let

$$
\phi_{k}(t)=t^{k}, k=0,1,2 .
$$


Then, the lower approximate solution and the upper approximate solution are

$$
\begin{aligned}
& \underline{y}_{\alpha}(t)=\underline{\theta}_{0}+\underline{\theta}_{1} t+\underline{\theta}_{2} t^{2}, \\
& \bar{y}_{\alpha}(t)=\bar{\theta}_{0}+\bar{\theta}_{1} t+\bar{\theta}_{2} t^{2} .
\end{aligned}
$$

From the fuzzy differential equation (3.10),

$$
\begin{aligned}
& \underline{y}_{\alpha}^{\prime \prime}(t)-\underline{y}_{\alpha}(t)=0 \Rightarrow-\underline{\theta}_{0}-\underline{\theta}_{1} t+\underline{\theta}_{2}\left(2-t^{2}\right)=0, \\
& \bar{y}_{\alpha}^{\prime \prime}(t)-\bar{y}_{\alpha}(t)=0 \Rightarrow-\bar{\theta}_{0}-\bar{\theta}_{1} t+\bar{\theta}_{2}\left(2-t^{2}\right)=0
\end{aligned}
$$

and from the boundary conditions (3.11),

$$
\begin{gathered}
\underline{y}_{\alpha}(0)=\underline{\theta}_{0}=1+\frac{1}{2} \alpha, \\
\bar{y}_{\alpha}(0)=\bar{\theta}_{0}=2-\frac{1}{2} \alpha, \\
\underline{y}_{\alpha}\left(\frac{3 \pi}{2}\right)=\underline{\theta}_{0}+\underline{\theta}_{1} \frac{3 \pi}{2}+\underline{\theta}_{2} \frac{9 \pi^{2}}{4}=3+\frac{1}{2} \alpha, \\
\bar{y}_{\alpha}\left(\frac{3 \pi}{2}\right)=\bar{\theta}_{0}+\bar{\theta}_{1} \frac{3 \pi}{2}+\bar{\theta}_{2} \frac{9 \pi^{2}}{4}=4-\frac{1}{2} \alpha .
\end{gathered}
$$

Taking $t=\frac{1}{2}$, from the equations (3.16), (3.18) and (3.20),

$$
\begin{gathered}
-\underline{\theta}_{1} \frac{1}{2}+\underline{\theta}_{2} \frac{7}{4}=1+\frac{1}{2} \alpha, \\
\underline{\theta}_{1} \frac{3 \pi}{2}+\underline{\theta}_{2} \frac{9 \pi^{2}}{4}=2
\end{gathered}
$$

are obtained. From here, the coefficients $\underline{\theta}_{1}, \underline{\theta}_{2}$ are obtained as

$$
\begin{aligned}
& \underline{\theta}_{1}=-0.9667505819-0.57381463442 \alpha, \\
& \underline{\theta}_{2}=0.29521411946+0.12176724731 \alpha .
\end{aligned}
$$

Also, from the equations (3.17), (3.19) and (3.21),

$$
\begin{gathered}
-\bar{\theta}_{1} \frac{1}{2}+\bar{\theta}_{2} \frac{7}{4}=3+\frac{1}{2} \alpha \\
\bar{\theta}_{1} \frac{3 \pi}{2}+\bar{\theta}_{2} \frac{9 \pi^{2}}{4}=2
\end{gathered}
$$

are obtained. From here, the coefficients $\bar{\theta}_{1}, \bar{\theta}_{2}$ are obtained as

$$
\begin{aligned}
& \bar{\theta}_{1}=-2.1143798507+0.57381463442 \alpha, \\
& \bar{\theta}_{2}=0.53874861409-0.12176724731 \alpha .
\end{aligned}
$$

Substituting these values in (3.14) and (3.15), the lower approximate solution and the upper approximate solution are obtained as

$$
\underline{y}_{\alpha}(t)=(1+0.5 \alpha)+(-0.9667505819-0.57381463442 \alpha) t
$$




$$
\begin{aligned}
& +(0.29521411946+0.12176724731 \alpha) t^{2} \\
\bar{y}_{\alpha}(t)= & (2-0.5 \alpha)+(-2.1143798507+0.57381463442 \alpha) t \\
& +(0.53874861409-0.12176724731 \alpha) t^{2}
\end{aligned}
$$

The approximate lower and upper solutions for $t=0.01$ are

$$
\begin{aligned}
& \underline{y}_{\alpha}(t)=0.99036201559+0.49426307132 \alpha, \\
& \bar{y}_{\alpha}(t)=1.97891007635-0.49427403038 \alpha .
\end{aligned}
$$

From the equations (3.12) and (3.13), the exact lower and upper solution for $t=0.01$ are

$$
\begin{gathered}
\underline{Y}_{\alpha}(t)=0.9905872695+0.4951139515 \alpha, \\
\bar{Y}_{\alpha}(t)=1.9808151724-0.49511395143 \alpha .
\end{gathered}
$$

Comparison the results of the lower exact solution and the lower approximate solution
$\alpha \quad \underline{Y}_{\alpha}(t)$
$0 \quad 0.9905872695$
$\underline{y}_{\alpha}(t)$
Error
$0.1 \quad 1.04009866465$
0.99036201559
0.00022525391
$0.2 \quad 1.0896100598$
1.03978832272
0.00031034193
$0.3 \quad 1.13912145495$
1.08921462985
0.00039542995
$\begin{array}{ll}0.4 & 1.1886328501\end{array}$
1.13864093698
0.00048051797
$\begin{array}{ll}0.5 & 1.23814424525\end{array}$
1.18806724411
0.00056533599
$0.6 \quad 1.2876556404$
1.23749355125
0.000650694
$\begin{array}{ll}0.7 & 1.33716703555\end{array}$
1.28691985838
0.00073578202
$\begin{array}{ll}0.8 & 1.3866784307\end{array}$
1.33634616551
0.00082087004
$0.9 \quad 1.43618982585$
1.38577247264
0.00090595806
1.43519877977
0.00099104608
$1 \quad 1.485701221$
1.48462508691
0.00107613409

Comparison the results of the upper exact solution and the upper approximate solution
$\alpha \quad \bar{Y}_{\alpha}(t)$
$\bar{y}_{\alpha}(t)$
Error
$0 \quad 1.9808151724$
1.97891007635
0.00190509605
$0.1 \quad 1.93130377726$
1.92948267332
0.00182110394
$0.2 \quad 1.88179238212$
1.88005527028
0.00173711184
$\begin{array}{ll}0.3 & 1.83228098698\end{array}$
1.83062786724
0.00165311974
$\begin{array}{ll}0.4 & 1.1886328501\end{array}$
1.7812004642
0.00156912763
$\begin{array}{ll}0.5 & 1.78276959183\end{array}$
1.73177306116
0.00148513553
$\begin{array}{ll}0.6 & 1.73325819669\end{array}$
1.68234565813
0.00140114342
$0.7 \quad 1.68374680155$
1.63291825509
0.00131715131
$0.8 \quad 1.58472401126$
1.58349085205
0.00123315921
$\begin{array}{cc}0.9 & 1.53521261612 \\ 1 & 1.48570122097\end{array}$
1.53406344901
0.00114916711
1.48463604597
0.001065175 


\subsection{The case of negative constant coefficient}

Consider the fuzzy boundary value problem

$$
\begin{gathered}
y^{\prime \prime}(t)=-\lambda y(t), \\
y(0)=A, \quad y(\ell)=B,
\end{gathered}
$$

where $\lambda>0$.

\subsubsection{The Exact Solution with Hukuhara Differentiability}

From the fuzzy differential equation (3.22), we have differential equations

$$
\begin{aligned}
& \underline{Y}_{\alpha}^{\prime \prime}(t)=-\lambda \bar{Y}_{\alpha}(t), \\
& \bar{Y}_{\alpha}^{\prime \prime}(t)=-\lambda \underline{Y}_{\alpha}(t)
\end{aligned}
$$

by using the Hukuhara differentiability and the fuzzy arithmetic property $-\left[\underline{Y}_{\alpha}, \bar{Y}_{\alpha}\right]$ $=\left[-\bar{Y}_{\alpha},-\underline{Y}_{\alpha}\right]$. Then, the lower solution and the upper solution of the fuzzy differential equation (3.22) are

$$
\begin{gathered}
\underline{Y}_{\alpha}(t)=-c_{1}(\alpha) e^{\sqrt{\lambda} t}-c_{2}(\alpha) e^{-\sqrt{\lambda} t}+c_{3}(\alpha) \sin (\sqrt{\lambda} t)+c_{4}(\alpha) \cos (\sqrt{\lambda} t), \\
\bar{Y}_{\alpha}(t)=c_{1}(\alpha) e^{\sqrt{\lambda} t}+c_{2}(\alpha) e^{-\sqrt{\lambda} t}+c_{3}(\alpha) \sin (\sqrt{\lambda} t)+c_{4}(\alpha) \cos (\sqrt{\lambda} t) .
\end{gathered}
$$

Using the boundary conditions (3.23), the coefficient $c_{1}(\alpha), c_{2}(\alpha), c_{3}(\alpha), c_{4}(\alpha)$ are obtained as

$$
\begin{gathered}
c_{1}(\alpha)=\left(\frac{1-\alpha}{2}\right)\left(\frac{(\bar{b}-\underline{b})-(\bar{a}-\underline{a}) e^{-\sqrt{\lambda} \ell}}{e^{\sqrt{\lambda} \ell}-e^{-\sqrt{\lambda} \ell}}\right), \\
c_{2}(\alpha)=\left(\frac{1-\alpha}{2}\right)\left((\bar{a}-\underline{a})-\frac{(\bar{b}-\underline{b})-(\bar{a}-\underline{a}) e^{-\sqrt{\lambda} \ell}}{e^{\sqrt{\lambda} \ell}-e^{-\sqrt{\lambda} \ell}}\right), \\
c_{3}(\alpha)=\frac{(\bar{b}+\underline{b})-(\bar{a}+\underline{a}) \cos (\sqrt{\lambda} \ell)}{2 \sin (\sqrt{\lambda} \ell)}, \\
c_{4}(\alpha)=\frac{\bar{a}+\underline{a}}{2} .
\end{gathered}
$$




\subsubsection{The Approximate Solution with the Undetermined Fuzzy Coefficients Method}

The undetermined fuzzy coefficients method is to seek an approximate solution as

$$
\tilde{y}_{N}(t)=\sum_{k=0}^{N} \tilde{\theta}_{k} \phi_{k}(t),
$$

where, $\phi_{k}(t), k=0,1, \ldots, N$ are positive basic functions whose all differentiations are positive and and the lower solution and upper solution are

$$
\underline{y}_{\alpha}(t)=\sum_{k=0}^{N} \underline{\theta}_{k}(\alpha) \phi_{k}(t), \quad \bar{y}_{\alpha}(t)=\sum_{k=0}^{N} \bar{\theta}_{k}(\alpha) \phi_{k}(t),
$$

respectively. Substituting the equation (3.24) in the fuzzy differential equation (3.22) and using the fuzzy arithmetic property $-\left[\underline{y}_{\alpha}, \bar{y}_{\alpha}\right]=\left[-\bar{y}_{\alpha},-\underline{y}_{\alpha}\right]$ yields

$$
\begin{aligned}
& \sum_{k=0}^{N} \underline{\theta}_{k}(\alpha) \phi_{k}^{\prime \prime}(t)+\lambda \sum_{k=0} \bar{\theta}_{k}(\alpha) \phi_{k}(t)=0, \\
& \sum_{k=0}^{N} \bar{\theta}_{k}(\alpha) \phi_{k}^{\prime \prime}(t)+\lambda \sum_{k=0}^{N} \underline{\theta}_{k}(\alpha) \phi_{k}(t)=0 .
\end{aligned}
$$

Using the boundary conditions (3.23),

$$
\begin{aligned}
& \sum_{k=0}^{N} \underline{\theta}_{k}(\alpha) \phi_{k}(0)=\underline{a}+\left(\frac{\bar{a}-\underline{a}}{2}\right) \alpha, \\
& \sum_{k=0}^{N} \underline{\theta}_{k}(\alpha) \phi_{k}(\ell)=\underline{b}+\left(\frac{\bar{b}-\underline{b}}{2}\right) \alpha, \\
& \sum_{k=0}^{N} \bar{\theta}_{k}(\alpha) \phi_{k}(0)=\bar{a}-\left(\frac{\bar{a}-\underline{a}}{2}\right) \alpha, \\
& \sum_{k=0}^{N} \bar{\theta}_{k}(\alpha) \phi_{k}(\ell)=\bar{b}-\left(\frac{\bar{b}-\underline{b}}{2}\right) \alpha
\end{aligned}
$$

are obtained. Taking

$$
\phi_{k}^{\prime \prime}(t)=\rho_{k}, \quad \lambda \phi_{k}(t)=\varepsilon_{k}, \quad \phi_{k}(0)=\zeta_{0 k}, \quad \phi_{k}(\ell)=\xi_{\ell k},
$$

we obtain

$$
\sum_{k=0}^{N} \underline{\theta}_{k}(\alpha) \rho_{k}+\sum_{k=0}^{N} \bar{\theta}_{k}(\alpha) \varepsilon_{k}=0
$$




$$
\begin{aligned}
& \sum_{k=0}^{N} \underline{\theta}_{k}(\alpha) \zeta_{0 k}=\underline{a}+\left(\frac{\bar{a}-\underline{a}}{2}\right) \alpha \\
& \sum_{k=0}^{N} \underline{\theta}_{k}(\alpha) \xi_{\ell k}=\underline{b}+\left(\frac{\bar{b}-\underline{b}}{2}\right) \alpha \\
& \sum_{k=0}^{N} \bar{\theta}_{k}(\alpha) \rho_{k}+\sum_{k=0}^{N} \underline{\theta}_{k}(\alpha) \varepsilon_{k}=0 \\
& \sum_{k=0}^{N} \bar{\theta}_{k}(\alpha) \zeta_{0 k}=\bar{a}-\left(\frac{\bar{a}-\underline{a}}{2}\right) \alpha \\
& \sum_{k=0}^{N} \bar{\theta}_{k}(\alpha) \xi_{\ell k}=\bar{b}-\left(\frac{\bar{b}-\underline{b}}{2}\right) \alpha
\end{aligned}
$$

Let us write the equations (3.25) - (3.30) as

$$
S(t) X(\alpha)=Y(\alpha),
$$

where

$$
\begin{gathered}
S=\left(\begin{array}{cc}
S_{1} & S_{2} \\
S_{2} & S_{1}
\end{array}\right), \\
S_{1}=\left(\begin{array}{cccc}
\rho_{1} & \rho_{2} & \ldots & \rho_{N} \\
\zeta_{00} & \zeta_{01} & \ldots & \zeta_{0 N} \\
\xi_{\ell 0} & \xi_{\ell 1} & \ldots & \xi_{\ell N}
\end{array}\right), \quad S_{2}=\left(\begin{array}{cccc}
\varepsilon_{1} & \varepsilon_{2} & \ldots & \varepsilon_{N} \\
0 & 0 & \ldots & 0 \\
0 & 0 & \ldots & 0
\end{array}\right), \\
X(\alpha)=\left(\underline{\theta}_{0} \underline{\theta}_{1} \ldots \underline{\theta}_{N}, \bar{\theta}_{0} \bar{\theta}_{1} \ldots \bar{\theta}_{N}\right)^{T}, Y(\alpha)=\left(0 \underline{A}_{\alpha} \underline{B}_{\alpha} 0 . \bar{A}_{\alpha} \bar{B}_{\alpha}\right)^{T} .
\end{gathered}
$$

From this, $\underline{\theta}_{0}, \underline{\theta}_{1}, \ldots \underline{\theta}_{N}, \bar{\theta}_{0}, \bar{\theta}_{1}, \ldots, \bar{\theta}_{N}$ are solved and the approximate solution is obtained.

Example 2. Consider the fuzzy boundary value problem

$$
\begin{gathered}
y^{\prime \prime}(t)=-y(t), t \in\left(0, \frac{3}{2} \pi\right), \\
y(0)=\left[1+\frac{1}{2} \alpha, 2-\frac{1}{2} \alpha\right], y\left(\frac{3 \pi}{2}\right)=\left[3+\frac{1}{2} \alpha, 4-\frac{1}{2} \alpha\right] .
\end{gathered}
$$

Using the Hukuhara differentiability and using the fuzzy arithmetic property $-\left[\underline{y}_{\alpha}, \bar{y}_{\alpha}\right]=\left[-\bar{y}_{\alpha},-\underline{y}_{\alpha}\right]$ in the fuzzy differential equation (3.31), we have

$$
\begin{aligned}
& \underline{Y}_{\alpha}^{\prime \prime}(t)+\bar{Y}_{\alpha}(t)=0, \\
& \bar{Y}_{\alpha}^{\prime \prime}(t)+\underline{Y}_{\alpha}^{\prime \prime}(t)=0 .
\end{aligned}
$$


Then, using the boundary conditions (3.32), the lower exact solution and the upper exact solution of the fuzzy differential equation (3.31) are obtained as

$$
\begin{aligned}
& \underline{Y}_{\alpha}(t)=\left(\frac{1-\alpha}{2}\right)\left(\frac{e^{-\frac{3 \pi}{2}}-1}{e^{\frac{3 \pi}{2}}-e^{-\frac{3 \pi}{2}}}\right) e^{t}+\left(\frac{1-\alpha}{2}\right)\left(\frac{1-e^{\frac{3 \pi}{2}}}{e^{\frac{3 \pi}{2}}-e^{-\frac{3 \pi}{2}}}\right) e^{-t}-\frac{7}{2} \sin (t)+\frac{3}{2} \cos (t), \\
& \bar{Y}_{\alpha}(t)=\left(\frac{1-\alpha}{2}\right)\left(\frac{1-e^{-\frac{3 \pi}{2}}}{e^{\frac{3 \pi}{2}}-e^{-\frac{3 \pi}{2}}}\right) e^{t}+\left(\frac{1-\alpha}{2}\right)\left(\frac{e^{\frac{3 \pi}{2}}-1}{e^{\frac{3 \pi}{2}}-e^{-\frac{3 \pi}{2}}}\right) e^{-t}-\frac{7}{2} \sin (t)+\frac{3}{2} \cos (t) .
\end{aligned}
$$

Let

$$
\phi_{k}(t)=t^{k}, k=0,1,2 .
$$

Then, the the lower approximate solution and the upper approximate solution are

$$
\begin{aligned}
& \underline{y}_{\alpha}(t)=\underline{\theta}_{0}+\underline{\theta}_{1} t+\underline{\theta}_{2} t^{2}, \\
& \bar{y}_{\alpha}(t)=\bar{\theta}_{0}+\bar{\theta}_{1} t+\bar{\theta}_{2} t^{2} .
\end{aligned}
$$

Substituting these equations in (3.31) and using $-\left[\underline{y}_{\alpha}, \bar{y}_{\alpha}\right]=\left[-\bar{y}_{\alpha},-\underline{y}_{\alpha}\right]$,

$$
\begin{aligned}
& \underline{y}_{\alpha}^{\prime \prime}(t)+\bar{y}_{\alpha}(t)=0 \Rightarrow \bar{\theta}_{0}+\bar{\theta}_{1} t+\bar{\theta}_{2} t^{2}+2 \underline{\theta}_{2}=0, \\
& \bar{y}_{\alpha}^{\prime \prime}(t)+\underline{y}_{\alpha}(t)=0 \Rightarrow \underline{\theta}_{0}+\underline{\theta}_{1} t+\underline{\theta}_{2} t^{2}+2 \bar{\theta}_{2}=0
\end{aligned}
$$

are obtained. From the boundary conditions (3.32), it yields

$$
\begin{gathered}
\underline{y}_{\alpha}(0)=\underline{\theta}_{0}=1+\frac{1}{2} \alpha, \\
\underline{y}_{\alpha}\left(\frac{3 \pi}{2}\right)=\underline{\theta}_{0}+\underline{\theta}_{1} \frac{3 \pi}{2}+\underline{\theta}_{2} \frac{9 \pi^{2}}{4}=3+\frac{1}{2} \alpha, \\
\bar{y}_{\alpha}(0)=\bar{\theta}_{0}=2-\frac{1}{2} \alpha, \\
\bar{y}_{\alpha}\left(\frac{3 \pi}{2}\right)=\bar{\theta}_{0}+\bar{\theta}_{1} \frac{3 \pi}{2}+\bar{\theta}_{2} \frac{9 \pi^{2}}{4}=4-\frac{1}{2} \alpha .
\end{gathered}
$$

Taking $t=\frac{1}{2}$, the equations

$$
\begin{gathered}
\bar{\theta}_{1} \frac{1}{2}+\bar{\theta}_{2} \frac{1}{4}+2 \underline{\theta}_{2}=-1-\frac{1}{2} \alpha, \\
\underline{\theta}_{1} \frac{3 \pi}{2}+\underline{\theta}_{2} \frac{9 \pi^{2}}{4}=2, \\
\underline{\theta}_{1} \frac{1}{2}+\underline{\theta}_{2} \frac{1}{4}+2 \bar{\theta}_{2}=-2+\frac{1}{2} \alpha, \\
\bar{\theta}_{1} \frac{3 \pi}{2}+\bar{\theta}_{2} \frac{9 \pi^{2}}{4}=2
\end{gathered}
$$


are obtained. From this, we have the coefficients $\underline{\theta}_{1}, \underline{\theta}_{2}, \bar{\theta}_{1}, \bar{\theta}_{2}$ as

$$
\begin{gathered}
\underline{\theta}_{1}=-74.981076422-0.5738146367 \alpha, \\
\underline{\theta}_{2}=16.001541876+0.1217672478 \alpha, \\
\bar{\theta}_{1}=-75.653915728+0.5738146324 \alpha, \\
\bar{\theta}_{2}=16.144322811-0.1217672469 \alpha .
\end{gathered}
$$

Substituting these values in the equations (3.35) and (3.36), the approximate lower and upper solutions

$$
\begin{aligned}
\underline{y}_{\alpha}(t)= & (1+0.5 \alpha)+(-74.981076422-0.5738146367 \alpha) t \\
& +(16.001541876+0.1217672478 \alpha) t^{2}, \\
\bar{y}_{\alpha}(t)= & (2-0.5 \alpha)+(-75.653915728+0.5738146324 \alpha) t \\
& +(16.144322811-0.1217672469 \alpha) t^{2}
\end{aligned}
$$

are obtained. The approximate lower and upper solutions for $t=0.01$ are

$$
\begin{gathered}
\underline{y}_{\alpha}(t)=0.2517893899+0.4942740304 \alpha, \\
\bar{y}_{\alpha}(t)=1.245075275-0.4942740304 \alpha .
\end{gathered}
$$

From the equations (3.33) and (3.34), the exact lower and upper solution for $t=0.01$ are

$$
\begin{aligned}
& \underline{Y}_{\alpha}(t)=0.9698116325+0.4951139514 \alpha, \\
& \bar{Y}_{\alpha}(t)=1.9600395353-0.4951139514 \alpha .
\end{aligned}
$$

Comparison the results of the lower exact solution and the lower approximate solution
$\alpha \quad \underline{Y}_{\alpha}(t)$
$\underline{y}_{\alpha}(t)$
Error
$\begin{array}{llll}0 & 0.9698116325 & 0.2517893899 & 0.7180222426\end{array}$
$\begin{array}{lllll}0.1 & 1.0193230276 & 0.3012167929 & 0.7181062347\end{array}$
$\begin{array}{lllll}0.2 & 1.0688344228 & 0.350644196 & 0.7181902268\end{array}$
$\begin{array}{llll}0.3 & 1.1183458179 & 0.400071599 & 0.7182742189\end{array}$
$\begin{array}{lllll}0.4 & 1.1678572131 & 0.4494990021 & 0.718358211\end{array}$
$\begin{array}{lllll}0.5 & 1.2173686082 & 0.4989264051 & 0.7184422031\end{array}$
$\begin{array}{lllll}0.6 & 1.2668800033 & 0.5483538081 & 0.7185261952\end{array}$
$\begin{array}{lllll}0.7 & 1.3163913985 & 0.5977812112 & 0.7186101873\end{array}$
$\begin{array}{lllll}0.8 & 1.3659027936 & 0.6472086142 & 0.7186941794\end{array}$
$\begin{array}{lllll}0.9 & 1.4154141888 & 0.6966360173 & 0.7187781715\end{array}$
$\begin{array}{lllll}1 & 1.4649255839 & 0.7460634203 & 0.7188621636\end{array}$ 


\section{Comparison the results of the upper exact solution and the upper approximate solution}

$\begin{array}{cccc}\alpha & \bar{Y}_{\alpha}(t) & \bar{y}_{\alpha}(t) & \text { Error } \\ 0 & 1.9600395353 & 1.245075275 & 0.7149642603 \\ 0.1 & 1.9105281402 & 1.195647872 & 0.7148802682 \\ 0.2 & 1.861016745 & 1.1462204689 & 0.7147962761 \\ 0.3 & 1.8115053499 & 1.0967930659 & 0.714712284 \\ 0.4 & 1.7619939547 & 1.0473656628 & 0.7146282919 \\ 0.5 & 1.7124825596 & 0.9979382598 & 0.7145442998 \\ 0.6 & 1.6629711645 & 0.9485108568 & 0.7144603077 \\ 0.7 & 1.6134597693 & 0.8990834537 & 0.7143763156 \\ 0.8 & 1.5639483742 & 0.8496560507 & 0.7142923235 \\ 0.9 & 1.514436979 & 0.8002286476 & 0.7142083314 \\ 1 & 1.4649255839 & 0.7508012446 & 0.7141243393\end{array}$

\section{Conclusions}

In this paper, the approximate solutions by using the undetermined fuzzy coefficients method and the exact solutions by using the Hukuhara differentiability of second-order fuzzy linear boundary value problems with positive and negative constant coefficients are investigated. The values of the exact and approximate solutions are computed for each $\alpha=0,0.1,0,2,0,3,0.4,0.5,0.6,0.7,0.8,0.9,1$. The error values between the exact and approximate solutions are found. Thus, comparison results of the found solutions are given. While the error values increase for the lower solutions of the worked problems, the error values decrease for the upper solutions. But, the error values for negative constant coefficient case are the more than the errors values for the positive constant coefficient case.

\section{REFERENCES}

[1] S. Abbasbandy and T. Allahviranloo, "Numerical solutions of fuzzy differential equations by Taylor method," Computational Methods in Applied Mathematics, vol. 2, no. 2, pp. 113-124, 2002, doi: 10.2478/cmam-2002-0006.

[2] S. Abbasbandy, T. Allahviranloo, O. Lopez-Pouso, and J. J. Nieto, "Numerical methods for fuzzy differential inclusions," Computers and Mathematics with Applications, vol. 48, no. 10-11, pp. 1633-1641, 2004, doi: 10.1016/j.camwa.2004.03.009.

[3] T. Allahviranloo, E. Ahmady, and N. Ahmady, "Nth-order fuzzy linear differential equations," Information Sciences, vol. 178, no. 5, pp. 1309-1324, 2008, doi: 10.1016/j.ins.2007.10.013.

[4] T. Allahviranloo, N. Ahmady, and E. Ahmady, "Numerical solution of fuzzy differential equations by predictor-corrector method," Information Sciences, vol. 177, no. 7, pp. 1633-1647, 2007, doi: 10.1016/j.ins.2006.09.015.

[5] B. Bede, "Note on "Numerical solutions of fuzzy differential equations by predictorcorrector method"," Information Sciences, vol. 178, no. 7, pp. 1917-1922, 2008, doi: 10.1016/j.ins.2007.11.016. 
[6] B. Bede and S. G. Gal, "Generalizations of the differentiability of fuzzy-number-valued functions with applications to fuzzy differential equations," Fuzzy Sets and Systems, vol. 151, no. 3, pp. 581-599, 2005, doi: 10.1016/j.fss.2004.08.001.

[7] J. J. Buckley and T. Feuring, "Fuzzy differential equations," Fuzzy Sets and Systems, vol. 110, no. 1, pp. 43-54, 2000, doi: 10.1016/S0165-0114(98)00141-9.

[8] J. J. Buckley and T. Feuring, "Fuzzy initial value problem for Nth-order linear differential equations," Fuzzy Sets and Systems, vol. 121, no. 2, pp. 247-255, 2001, doi: 10.1016/S01650114(00)00028-2.

[9] D. Dubois and H. Prade, "Operations on fuzzy numbers," International Journal of Systems Science, vol. 9, no. 6, pp. 613-626, 1978, doi: 10.1080/00207727808941724.

[10] N. Gasilov, S. E. Amrahov, and A. G. Fatullayev, "A geometric approach to solve fuzzy linear systems of differential equations," Applied Mathematics and Information Science, vol. 5, no. 3, pp. 484-499, 2011.

[11] X. Guo, D. Shang, and X. Lu, "Fuzzy approximate solutions of second-order fuzzy linear boundary value problems," Boundary Value Problems, 2013, doi: 10.1186/1687-2770-2013-212.

[12] O. He and W. Yi, "On fuzzy differential equations," Fuzzy Sets and Systems, vol. 32, no. 3, pp. 321-325, 1989, doi: 10.1016/0165-0114(89)90264-9.

[13] E. Hüllermeir, "An approach to modelling and simulation of uncertain dynamical systems," International Journal of Uncertainty, Fuzziness and Knowledge-Based Systems, vol. 5, no. 2, pp. 117-137, 1997, doi: 10.1142/S0218488597000117.

[14] O. Kaleva, "Fuzzy differential equations," Fuzzy Sets and Systems, vol. 24, no. 3, pp. 301-317, 1987, doi: 10.1016/0165-0114(87)90029-7.

[15] A. Khastan and J. J. Nieto, "A boundary value problem for second order fuzzy differential equations," Nonlinear Analysis, vol. 72, no. 9-10, pp. 3583-3593, 2010, doi: 10.1016/j.na.2009.12.038.

[16] H.-K. Liu, "Comparison results of two-point fuzzy boundary value problems," International Journal of Computational and Mathematical Sciences, vol. 5, no. 1, pp. 1-7, 2011.

[17] M. L. Puri and D. A. Ralescu, "Differentials of fuzzy functions," Journal of Mathematical Analysis and Applications, vol. 91, no. 2, pp. 552-558, 1983, doi: 10.1016/0022-247X(83)90169-5.

Author's address

Hülya Gültekin Çitil

Giresun University, Department of Mathematics, Faculty of Arts and Sciences, 28100 Giresun, Turkey

E-mail address: hulya.citilegiresun.edu.tr 